\title{
JOURNAL OF ISTANBUL VETERINARY SCIENCES
}

\section{Protective immune studies against fungi}

\section{Aikerim Kumondorova ${ }^{1}$, Kemal Metiner ${ }^{2}$}

\section{Review Article}

Volume: 4, Issue: 3 December 2020

Pages: 96-101

\section{Article History}

Received: 10.08 .2020

Accepted: 17.10.2020

Available online:

22.10.2020
1. Istanbul University Cerrahpasa, Institute of Graduate Studies, 34320, Avcilar/ Istanbul, Turkey. 2. Istanbul University-Cerrahpasa, Faculty of Veterinary Medicine, Department of Microbiology, Buyukcekmece Campus 34500, Istanbul, Turkey. Kumondorova, A. ORCID: 0000-0001-7341-1597, Metiner, K. ORCID: 0000-0003-4105-5852.

\section{ABSTRACT}

The immune system is the host's defence against different agents and infections. Understanding the complex and highly dynamic interactions between fungi and host cells in a tissue-specific manner is crucial to facilitate the development of new therapeutic approaches to infections. Generally fungal pathogens rarely cause diseases in immunologically competent individuals. However, commensal and non-pathogenic environmental fungi can cause life-threatening infections in individuals with immune deficiency. Understanding the molecular and cellular bases of immunity to fungi has progressed significantly over the past few years. Despite close interactions with fungi today, how the immune system protects humans and animals from fungal pathogens has not been fully elucidated compared to the immune response to bacteria or viruses. The immune system is the host's defence against various foreign proteins and infections. Understanding the complex and highly dynamic interactions between fungi and host cells is crucial for the development of new therapeutic approaches to infections. Researchers from 15 countries in Europe, Asia, Australia, North and South America have provided the last five years review and original research articles that consist of a wide range of fungal pathogens, disease, effector, regulatory cells and molecular pathways of host immune responses to fungal exposure. In this review, we summarize an outline of the recent findings, perspectives, and reviews about the complex and highly dynamic interactions between fungi and host cells and a contemporary understanding of protective immunity against fungi. This review will allow an overview of the most exciting recent advances in antifungal immunity, discoveries that will help pave the way for the development of new strategies that are seriously needed to combat these devastating diseases.

Keywords: adaptive immunity, innate immunity, fungal infections, host immune system

DOI: https://doi.org/10.30704/http-www-jivs-net.778761

To cite this article: Kumondorova, A., Metiner, K. (2020). Protective immune studies against fungi. Journal of Istanbul Veterinary Sciences, 4(3), 96-101. Abbreviated Title: J Ist Vet Sci

\section{Introduction}

Fungi are eukaryotic heterotrophs with potentially more than 5 million species that can be found in any environment. Although there are a vast number of fungi in the world, only a small number (about 300 species) can cause disease in humans and animals. The most successful pathogens among these share the ability to grow at the physiologic temperature of

\footnotetext{
*Corresponding Author: Aikerim Kumondorova

E-mail: akumondorova@gmail.com
}

endothermic vertebrates and consequently colonize or infect only susceptible hosts. Most of the diseasecausing fungi are opportunistic pathogens. They only cause disease under certain conditions - such as when the immune system becomes weakened (Templeton et al. 2018). Immune-competent humans and animals are mostly resistant to fungal infections that were

Journal home page: www.jivs.net http://dergipark.gov.tr/http-www-jivs-net 
investigated rare and remained imperfectly understood throughout much of life history. In any case, since 1980, the prevalence of opportunistic fungal diseases has steadily increased in parallel with increases in individuals with acquired immune deficiencies or those receiving immune suppressive or myeloablative therapies. For example, chemotherapy, immunosuppressive drugs, and HIV infection cause disruption of the immune system, which is stated by researchers, which means that fungi can infect these vulnerable patients more easily (Fisher et al., 2018).

Fungi can cause many different types of infections. These can result in widespread skin and mucosal infections, serious life-threatening systemic infections, sepsis and organ failure. In both cases, there is a limited number of treatments available and they do not currently have vaccines to prevent these infections. Therefore, there is a growing interest in studies on fungal biology and host-fungal interactions that can identify the immediate need for new treatments, new antifungal goals, or alternative. Continuous exposure of humans and animals to both commensal and environmental fungi require a strong immune system for tolerance and protection while limiting collateral damage caused by excessive or harmful inflammations has been reported by researchers (Bongomin et al., 2017).

\section{Fungal Infections and Immunity}

Fungi are common inhabitants of host barrier surfaces such as the oral cavity, skin, vagina, gut, lungs. And the immune system has co-evolved and adapted to their presence over millions of years. Changed immune status, usually due to treatment with immunosuppressive drugs or sometimes caused by inherited weaknesses in host defense, leads to increased susceptibility to different fungal infections. Fungi are associated with a wide variety of diseases in mammals, starting from cutaneous lesions and acute self-limiting pulmonary manifestations in immunecompetent individuals to inflammatory diseases and severe life-threatening infections in immunecompromised patients. Mucosal infections are more prevalent than invasive infections and are a major cause of morbidity. In contrast to bacterial and viral infections, an effective vaccine against fungal infections has not been developed yet. Currently, available antifungal drugs are only partly successful in treating invasive fungal infections (Campos et al., 2015).

Fungi infections with high contagiousness are caused by some fungal species in three opportunistic fungi. These three genera are Candida spp., Aspergillus spp., and Cryptococcus spp. These species can exist in two morphological forms: yeasts with different cell wall compositions (simultaneous reproduction with conidia formation asexual cell forms) and hyphae branching multicellular structures, tubular filaments). While hypha morphology is generally associated with tissue infestation, it is stated that the conidial form is associated with colonization, which suggests different host recognition mechanisms and explains the contrast in virulence (Kumar et al., 2018).

Researchers have found that Candida species remain the fourth most crucial factor in hospitalacquired bloodstream infections. However, invasive aspergillosis and other mold infections, mostly caused by Aspergillus fumigatus and $A$. terreus are the leading causes of infection-related deaths in patients with hematopoietic stem cell transplantation (Khanna et al., 2016).

In the past two decades, the immunopathogenesis of fungal infections has been described primarily in terms of Th1 / Th2 balance. Although the Th1 mediated defense guided by IL-12 / IFNg is at the center of immunity against fungi, other cytokines and $\mathrm{T}$ cell-dependent protection routes are now considered obsolete concepts. In the defense against fungi, due to new research, the immune response through Th17 can play a role in the formation of inflammation attributed to their uncontrolled responses, and chronic inflammatory events can be associated with recurrent fungal infections (Romani, 2008).

A recent study shows that host-specific receptors recognize fungal-specific ligands and activate signal cascades that initiate the phagocytosis of fungi, proinflammatory mediators, the formation of reactive oxygen reactions, the accumulation of inflammatory cells into the sites of infection and the activation of acquired immunity. A better understanding of the molecular mechanisms that form the basis of defense against fungi provides important infrastructure for the development of protective vaccines, therapeutic drugs, and research of basic strategies (Hohl et al., 2006).

Leib und Gut-Landmann et al. (2012), in their published article, focused on the recognition of fungi by the host immune system, concepts emerging in effector mechanisms, creating protective $\mathrm{T}$ cell responses and developing vaccine-based treatments for vulnerable patient groups, and analyzed the natural and adapted immune system against fungal pathogens. In their study, they also emphasized that IL $-1 \beta$ murine production is critical for host defense in mouse models exposed to candidiasis. Production and release of $\mathrm{IL}-1 \beta$ require two independent signals: one 
regulates the transcription and translation of pro-IL$1 \beta$, and the other causes its proteolytic cleavage to transform into active IL-1 $\beta$. Fungi trigger both stages of IL-1 $\beta$ synthesis by CLR-dependent caspase activation by combining inflammasomes with different subunit composition. The NOD-like receptor NLRP3 (Nucleotide oligomerization domain-like receptor family, pyrin domain containing 3 ) and the adaptor protein ASC (Apoptosis-associated speck-like protein containing a CARD) form the scaffold of the NLRP3 inflammasome for caspase-1-activation. Mice with NLRP3 and ASC deficiency have no caspase-1 activation and IL-1 $\beta$ secretion. In addition, it was noticed that increased mortality in response to Candida albicans infection as well. Moreover, in humans, allelic variations in NLRP3 have been associated with recurrent vulvovaginal candidiasis cases.

Khanna et al. (2016) have investigated how immunology and host genetics could help during fungal infections; especially they focused on Candida spp. and Aspergillus spp. invasions. They realized that genetic and immunological defects in innate and adaptive immune systems led to an increased risk of invasive fungal infections among those who received chemotherapy or transplant. These differences have been argued, in part, from the individual genetic makeup that will increase or decrease the susceptibility to infections. Based on this hypothesis, many researchers analyzed whether single nucleotide polymorphisms in genes involved in immune responses against fungal pathogens influenced susceptibility to infections. In summary, this study showed how genetic polymorphisms could predispose to the development of invasive fungal infections, especially in the signal pathways of innate immune cells.

Another study by Jiang (2016) published a review on immunology and immune genetics. The review focused on two main host immune responses (natural and adaptive) against fungi and large immune cells such as macrophages, neutrophils, dendritic cells, $T$ and $B$ cells. He tried to clarify the mechanical aspects of the antifungal effect of each cell type and how these cells and their mechanisms can support future vaccine strategies. He also stated that the natural and adaptive immune response against fungi is important, but remains poorly understood for today.

Furthermore, Uehling et al. (2017) published an article, which was named as "Do fungi have an innate immune response?" In this review, they tried to find answers about the biological interactions of fungi, especially between plants and animals. Scientists focused on fungi NLR proteins and how they may use similar mechanisms to recognize and respond to heterospecific species. The NOD-like receptors (NLRs) contribute to the recognition and discrimination process in plants and animals as well. As a result, they outlined of fungus similarities and differences with their plant and animal counterparts, and proposed future directions elucidating aspects of fungal immune systems. They noticed the evolutionary success of the kingdom Fungi and the diversity of fungal biotic interactions with plants and animals, how fungi had developed the ability to identify and respond to interacting organisms also. However, mechanisms for such monitoring and response are just beginning to be understood. Understanding NLR-mediated fungal immunity in pathogenic fungi reveals specific targets for drug development to activate fungal cell death.

Lionakis et al., (2017) published a study focusing on the contemporary understanding of protective immunity against fungi. This review is based on information from animal models and patients with primary immunodeficiency disorders (PIDD). In particular, these models were patients who did not have an allergic or toxin-mediated fungal disease. In addition, this study attempted to actively explain the fungal recognition mechanism and immune activation, together with an in-depth study of the fungal cell wall structure. The cell wall of the fungi contains polysaccharide and lipid layers that activate the immune system. The cell wall is located outside the plasma membrane, and this wall consists of several layers: the innermost layer consists of chitin, a $\mathrm{N}$ acetylglucosamine polymer. The external layer is formed by immune reactive $\beta-(1,3)$ and $\beta-(1,6)$ glucans, which are hidden by many fungi (Erwig \& Gow., 2016).

Also, these scientists have studied different relationships between various cell receptors such as Ctype lectin receptors, toll-like receptors, NOD-like receptors and other $\mathrm{CD} 14$ receptors. Together, they also studied various mechanisms in detail between the fungus and the host. They showed how the fungi enter the host cell and what processes will follow step by step. As a result, these scientists have demonstrated recent advances that the antifungal binding of the dectin1 / CARD9 (Caspase recruitment domain-containing protein 9) and IL-17 pathways in antifungal immunity. Another conclusion was that the discovery of $\beta$-glucan-induced trained immunity and conserved sterilizing immunity-mediating epitopes lays the foundation for clinical trials to test vaccine protection against multiple fungal genera and species (Garfoot et al., 2016). 
Recent studies have also shown in the article of Kumar et al., (2018). It stated that immunological and genetic studies had shown the crucial role of human immune disorders in fungal infections. In contrast to viral and antibacterial infections, experimental vaccine against fungal infections has not been developed yet, and available antifungal drugs are only partly successful in treating patients and animals with fungal infections. The research team also noticed the most invasive fungal infections such as Candida spp., Aspergillus spp., and Cryptococcus spp from three genera and analyzed in detail about their features. For instance, how they are evading host-induced programmed cell death. Especially, how the $A$. fumigatus expresses the gene AfBir1 during this kind of process, this gene is homologous to the human Survivin gene, which contains a BIR domain that is involved in the suppression of apoptosis by caspase inhibition and it was reported that these findings highlight the potential for identifying drug targets in the pathogen genome.

It was also mentioned about the human gut, which is present CLRs dectin- 1 and dectin- 3 are PRRs that are important in mediating anti-fungal responses to intestinal fungi. Upon colonization of mouse intestine with $C$. albicans, some fungal PRRs such as dectin-1, dectin2, and Mincle were highly expressed in gutresident CX3CR1+ mononuclear phagocytes (MNPs) than in dendritic cells. Specific diminution of CX3CR1+ MNPs in mice resulted in a decrease in anti-fungal Th17 cells and IgG antibody responses against intestinal C. albicans, not against systemic infection. These findings highlight the importance of tissuespecific cellular functions and tissue-specific cellular functions in fungal infections. It was also investigated the effect of genetic variations in the human CX3CR1 gene on immunity to fungal infections in patients with inflammatory bowel disease. The article mentioned about another interferon pathway, type III IFNs (IFN$\lambda s)$, as a crucial regulator of antifungal neutrophil responses against $A$. fumigatus (Leonardi et al., 2018).

Along with another study which named as "Immunity to Human Fungal Pathogens: Mechanisms of Host Recognition, Protection, Pathology, and Fungal Interference" by Templeton et al. (2018) was given a short brief about the mechanisms of immunity to human fungal pathogens. Additionally, they stated that early fungal recognition and inflammation provide critical signals that drive adaptive antifungal immunity. However, important variations encountered by the host innate immune system, and evolutionary adaptation of pathogenic fungi, drive diverse disease outcomes ranging from tolerance, clearance and resolution to dissemination and immense inflammation. They also report that the exposure of hyphae of the dematiaceous mold Curvularia lunata to human THP1 monocytes resulted in increased inflammatory IL-8 and regulatory IL-10, offering a possible mechanism for the proficiency of this species to cause chronic infections in immunecompetent individuals. Another article also says that hypha of the yeast $C$. albicans induced low levels of cytokine secretion from human monocytes, with the highest levels from yeast and intermediate levels from pseudohypha, and cell wall mannan depletion partially reversed these responses (Mukaremera et al., 2017).

It is important to mention a recent study by Freitas et al., (2019) about fungal extracellular vesicles as potential targets for immune interventions. The article reports how important the release of extracellular vesicles by fungi and about its basic cellular process. The scientists from this study stated that vesicles may might play a pivotal role in the establishment of fungal infections, as they can interact with the host immune system to bring out multiple outcomes. They observed that, depending on the fungal pathogen, extracellular vesicles could exacerbate or attenuate fungal infections. The research shows the interaction between fungal extracellular vesicles and the host immune system and how an understanding of the mechanisms that regulate those interactions might be useful for the development of new adjuvants as well as the improvement of protective immune responses against infectious or noninfectious diseases.

\section{Vaccination}

Despite today's medical need, there is no vaccine commercially used for opportunistic mycoses. Mutants, whose virulence is attenuated, represent one of the best ways to stimulate immunity and provide maximum protection, as evidenced by experimental models of blastomycosis, histoplasmosis and coccidioidomycosis (Wüthrich et al., 2011). Besides, vaccination with attenuated mutants induces antifungal memory CD8 + T cells, maintained for at least six months without numerical or functional loss (Nanjappa et al., 2012). While these vaccine strains are unlikely to be safe in people with weakened immunity, the information from experimental vaccine models is crucial for the rational design of human vaccine candidates in the future. The mechanisms of protecting experimental vaccines prepared against candidiasis, aspergillosis and endemic mycoses owe Th17 and Th1 cells, especially for human vaccines (Wüthrich et al., 2011). In summary, fungal vaccines protect by $T$ cell activation and antibodies. $T$ cell- 
based vaccines mediate immunity acquired by the production of inflammatory cytokines (IFN-y, TNF, IL17 and IL-22), which are killed by phagocytes and regulate the synthesis of epithelial cell-secreted antimicrobial proteins such as cathelicidines and histatins (Romani, 2011). Antibodies neutralize virulence factors (e.g., adhesins), inhibit the growth of fungi and contribute to protection by stimulating direct killing, opsonophagocytosis and complement activation. Thus, strategies using both ways of adaptive immune response have been reported to be the most rational and successful vaccine candidates (Huang et al., 2010).

Study by Ural and Ulutas focused on the Trichophyton verrucosum vaccine. To explain in detail, the immune response of animals was observed by naturally infecting the $T$. verrucosum agent to racehorses. The aim of this study was to investigate how effective the $T$. verrucosum vaccine against the horses infected by $T$. equinum. Cross immune effects in these animals have been observed. A total of 25 racehorses between the ages of 2 and 14 received random intramuscular lyophilized $T$. verrucosum vaccine. Clinical evaluations were recorded at the beginning, middle and end of the treatment. At the end of the study, the clinical picture was significantly reduced in vaccinated horses $(P<.001)$. The symptoms of trichophytosis in all horses vaccinated started to decrease gradually within 7 to 12 days after vaccination. Complete clinical remission was detected within 28 to 42 days, and all horses treated became culture negative within 25 days after starting treatment. No clinical improvement was observed in nine racehorses used for control purposes, which were never vaccinated throughout the study. It was found that any infection was not noticed in all horses that received the vaccine within ten months of vaccination. As a result, in this study, they stated that the inactive $T$. verrucosum vaccine creates a safe and effective immune response for racehorses infected by T. equinum (Ural and Ulutas., 2008).

In another study in Europe, the effectiveness of an inactive vaccine for the treatment of dermatophytosis in felines was investigated in detail via a controlleddouble-blind multicenter GCP study. Fifty-five cats with dermatophytosis confirmed by fungal culture, caused by Trichophyton mentagrophytes or Microsporum canis, were vaccinated intramuscularly. The vaccine was administered intramuscularly every two weeks. Clinical symptoms were evaluated on days 14, 28 and 42. Clinical symptoms were recorded and considered according to their severity. Whether the applied vaccine is working was analysed through the number of lesions decreased and the severity of the lesion decreased. The primary assessment point was made for cats under one-year-old and cats with the first infection. At this point, the effect of the vaccine applied to young cats was significantly more successful than placebo cats used for control purposes (total of lesions: $p=0.0446$; scored score $x$ number: $p$ $=0.0405)$. In cats with more severe lesions, the difference in vaccine administration for the second time was more pronounced. The affected exotic cats also improved using these parameters. Based on this study, the inactivated vaccine investigated stated that the clinical signs of dermatophytosis can be used as a treatment protocol to accelerate healing in younger severely affected cats and cats with initial infection (Westhoff et al., 2010).

\section{Conclusion}

Despite significant advances in our understanding of host immunity to fungal exposure and infection, the treatment of fungal diseases has not progressed beyond the use of a limited repertoire of antifungal drugs that are rendered increasingly ineffective by emerging fungal resistance. In conclusion, the recent studies described in original research and review articles in this survey provide a positive direction for the future of antifungal immune therapy. Present achievements by fungal immunologists have significantly increased our awareness of the basic mechanisms of innate and adaptive immunity, inflammation, regulation of antifungal immune responses at molecular, cell, tissue, and organismal levels. We hope these articles will stimulate further research in terms of novel antifungal therapies, with more investment in this research area now needed to stimulate interest in solving current and future challenges posed by a fungal disease.

\section{References}

Bongomin, F., Gago, S., Oladele, R. O., \& Denning, D. W. (2017). Global and multinational prevalence of fungal diseases-estimate precision. Journal of Fungi (Basel), 3:E57.

Campos, J. H., Soares, R. P., Ribeiro, K., Andrade, A.C., Batista, W. L., \& Torrecilhas, A. C. (2015). Extracellular vesicles: role in inflammatory responses and potential uses in vaccination in cancer and infectious diseases. Journal of Immunology Research, 2015:832057.

Freitas, M. S., Bonato, V. L. D., Pessoni, A. M., Rodrigues, M.L., Casadevall, A., \& Almeifa, F. (2019). 
Fungal extracellular vesicles as potential targets for immune interventions. Journal of Clinical Mycrobiology (M Sphere), 4:e00747-19.

Fisher, M. C., Hawkins, N. J., Sanglard, D., \& Gurr, S. J. (2018). Worldwide emergence of resistance to antifungal drugs challenges human health and food security. Science, 360, 739-42.

Erwig, L. P., \& Gow, N. A. (2016). Interactions of fungal pathogens with phagocytes. Nature Reviews Microbiology, 14(3),163-176.

Garfoot, A. L., Shen, Q., WXthrich, M., Klein, B. S., \& Rappleye, C. A. (2016). The Eng1 $\beta$-glucanase enhances histoplasma virulence by reducing $\beta$ glucan exposure. American Society for Microbiology, MBio; 7(2):e01388-e01315.

Huang, H., Ostroff, G. R., Lee, C. K., Specht, C. A., \& Levitz, S. M. (2010). Robust stimulation of humoral and cellular immune responses following vaccination with antigen-loaded beta-glucan particles. MBio, 1(3):e00164.

Khanna, N., Stuehler, C., Lünemann, A., Agnieszka, W., Pierre-Yves B., \& LeibundGut-Landmann, S. (2016). Host response to fungal infections-how immunology and host genetics could help to identify and treat patients at risk. Swiss Medical Weekly, 21, 146:w14350.

Kumar, V., van de Veerdonk, F.L., \& Netea, M. G. (2018). Antifungal immune responses: emerging host-pathogen interactions and translational implications. Genome Medicine, 10:39.

LeibundGut-Landmann, S., Wüthrich, M., \& Hohl, T. M. (2012). Immunity to Fungi. Current opinion in immunology, 24(4), 449-458.

Leonardi, I., Li, X., Semon, A., Li, D., Doron, I., Putzel, G., Bar, A., Rescigno, M., McGovern, D. P. B., Pla, J., \& Iliev, D. I. (2018). CX3CR1+ mononuclear phagocytes control immunity to intestinal fungi. Science. 359, 232-236.

Lionakis, M. S., Iliev, I. D., \& Hohl, T. M. (2017). Immunity against fungi. Journal of clinical investigation insight, 2(11):e93156.

Mukaremera, L., Lee, K. K., Mora-Montes, H. M., \& Gow, N. A. R. (2017). Candida albicans yeast, pseudohyphal, and hyphal morphogenesis differentially affects Immune recognition. Front Immunol, 7, 8:629.

Nanjappa, S. G., Heninger, E., Wüthrich, M., Sullivan, T., \& Klein, B. (2012). Protective antifungal memory $\mathrm{CD} 8+\mathrm{T}$ cells are maintained in the absence of CD4+ $T$ cell help and cognate antigen in mice. Journal of Clinical Investigation, 122, 987-999.

Romani, L. (2008). Cell mediated immunity to fungi: a reassessment. Medical Mycology, 46(6), 515-529.

Romani, L. (2011). Immunity to fungal infections. Nature Reviews Immunology, 11, 275-288.

Jiang, S. (2016). Immunity against fungal infections. Immunology and immunogenetics insights, 8, 3-6.

Hohl, T. M., Rivera, A., \& Pamer, E. G. (2006). Immunity to fungi. Current Opinion in Immunology, 18(4), 465 -472 .

Templeton, S. P., Rivera, A., Hube, B., \& Jacobsen, I. D. (2018). Immunity to Human Fungal Pathogens: Mechanisms of host recognition, protection, pathology, and fungal interference. Frontiers in Immunology. 9:2337.

Uehling, J., Deveau, A., \& Paoletti, M. (2017). Do fungi have an innate immune response? An NLR-based comparison to plant and animal immune systems. PLoS Pathogens, 13(10): e1006578.

Ural, K., \& Ulutas. B. (2008). Immunization with Trichophyton verrucosum vaccine in hunter/Jumper and dressage horses with naturally occurring Trichophyton equinum infection: A Prospective, randomized, double-blinded, placebo-controlled clinical trial. Journal of Equine Veterinary Science, 28 (10),590-593.

Westhoff, D. K., Kloes, M. C., Orveillon, F. X., Farnow, D., Elbers, K., \& Mueller, R. C. (2010). Treatment of feline dermatophytosis with an inactivated fungal vaccine. The Open Mycology Journal, 4, 10-17.

Wüthrich, M., Hung, C. Y., Gern, B. H., Pick-Jacobs, J. C., Galles, K. J., Filutowicz, H. I., Cole, G. T., \& Klein, B. S. (2011). A TCR transgenic mouse reactive with multiple systemic dimorphic fungi. Journal of Immunology, 187, 1421-1431.

Wüthrich, M., Gern, B., Hung, C. Y., Ersland, K., Rocco, N., Pick-Jacobs, J., Galles, K., Filutowicz, H., Warner, T., Evans M., Cole, G., \& Klein, B. (2011). Vaccineinduced protection against 3 systemic mycoses endemic to North America requires Th17 cells in mice. Journal of Clinical Investigation, 121, 554-568. 